Supporting Information

\title{
Scalable Polymeric Few-Nanometer Organosilica Membranes with Hydrothermal Stability for Selective Hydrogen Separation
}

Lingxiang Zhu, ${ }^{\perp, \dagger, \sharp \S}$ Liang Huang, ${ }^{\perp, \dagger}$ Surendar R. Venna, ${ }^{\ddagger}$, Adrienne K. Blevins, $\|$ Yifu Ding,\|

David P. Hopkinson, ${ }^{\ddagger}$ Mark T. Swihart, ${ }^{\dagger}$ and Haiqing Lin ${ }^{* \dagger}$

$\uparrow$ Department of Chemical and Biological Engineering, University at Buffalo, The State

University of New York, Buffalo, NY 14260, USA

† U.S. Department of Energy, National Energy Technology Laboratory, 626 Cochrans Mill

Road, P.O. Box 10940, Pittsburgh, PA 15236, USA

$\S$ NETL Support Contractor, 626 Cochrans Mill Road, P.O. Box 10940, Pittsburgh, PA 15236, USA

\| Membrane Science, Engineering and Technology (MAST) Center, Paul M. Rady Mechanical

Engineering, University of Colorado at Boulder, Boulder, CO 80309, USA

$\perp$ These authors contributed equally to this work.

* Corresponding author: haiqingl@buffalo.edu (H.L.) 


\section{Supplementary results and discussion}

\section{Supplementary figures}

Figure S1 shows surface and cross-sectional micrographs of a PBI porous support film. The support has surface pores less than $20 \mathrm{~nm}$ in diameter. As shown in the cross-section image, this support has a finger structure and a thin microporous skin layer, providing very high gas permeance. At $23{ }^{\circ} \mathrm{C}$, it exhibits $\mathrm{H}_{2}$ and $\mathrm{CO}_{2}$ permeance of $\sim 400,000 \mathrm{GPU}$ and $\sim 100,000 \mathrm{GPU}$, respectively.
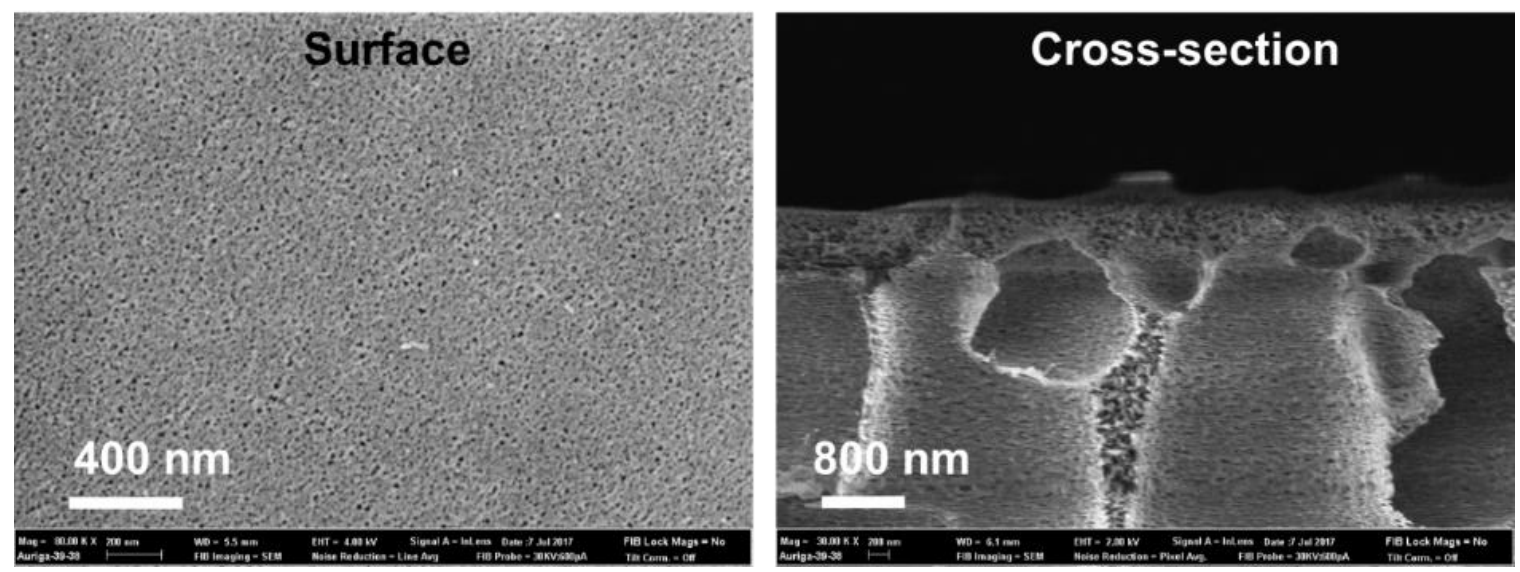

Figure S1. Surface and cross-section SEM images of a porous PBI support.

Figure S2 displays the surface roughness of the PDMS and POSi samples on the Si wafers. The AFM cantilever generates $20 \mathrm{~nm}$-deep indentation traces on the soft PDMS because of high adhesion forces ( $c f$. Figure S2a). Due to the formation of a silica surface layer with improved mechanical properties, POSi120 and POSi360 are free from indentation traces and show a smoother surface than PDMS.

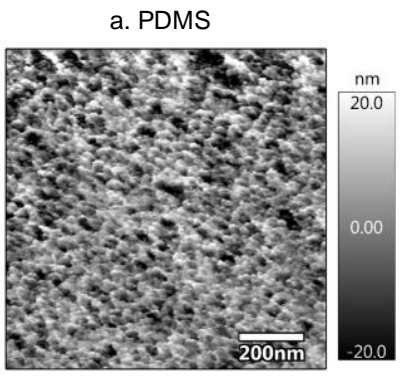

b. POSi120

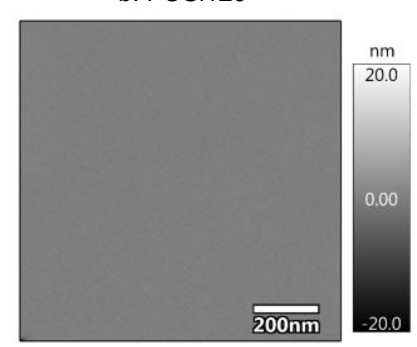

c. POSi360

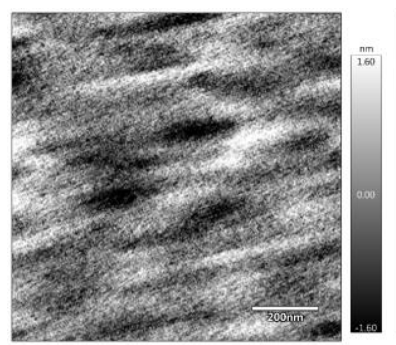

d. POSi360

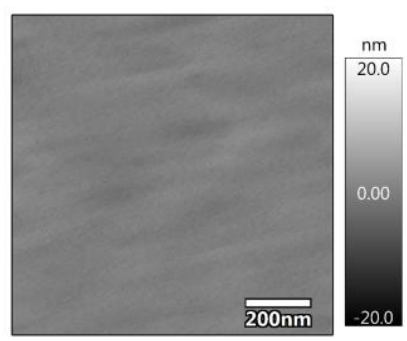

Figure S2. AFM images showing the surface roughness of a) PDMS, b) POSi120, and c) and d) POSi360 with the same height scale in a), b), and d), and a much smaller range of height in c).

Figure S3 compares unresolved O 1s XPS spectra of PDMS and the POSi samples. All values in $\mathrm{eV}$ are relative to $\mathrm{C} 1 \mathrm{~s}=284.6 \mathrm{eV}$. Oxygen element in PDMS only has one chemical state as Si$\mathrm{O}-\mathrm{Si}$ bond, presenting an $\mathrm{O} 1 \mathrm{~s}$ peak at $532.2 \mathrm{eV}$. After plasma treatment, $\mathrm{O} 1 \mathrm{~s}$ peaks for the POSi samples become broader, suggesting an increase in the number of chemical states of oxygen. C$\mathrm{O}-\mathrm{H}$ and $\mathrm{Si}-\mathrm{O}-\mathrm{H}$ bonds were reported to show characteristic peaks at $532.5 \pm 0.2 \mathrm{eV}$ and $533.3 \pm$ 
$0.3 \mathrm{eV}$, respectively. ${ }^{1,2}$ Considering $\mathrm{C}-\mathrm{O}-\mathrm{H}$ and $\mathrm{Si}-\mathrm{O}-\mathrm{H}$ binding energies are given in a wide range, accurate peak assignment is not possible in this case. However, it can be concluded that $\mathrm{Si}-\mathrm{O}-\mathrm{H}$ groups are formed after plasma treatment because $\mathrm{O}$ 1s peaks for all the three POSi samples shift to $532.8 \pm 0.1 \mathrm{eV}$. If only $\mathrm{C}-\mathrm{O}-\mathrm{H}$ groups were present, the $0.6 \mathrm{eV}$ shift would be impossible. The presence of C-O-H groups was already evidenced in the analysis of XPS C 1s spectra of PDMS and the POSi samples displayed in Figure 2c."

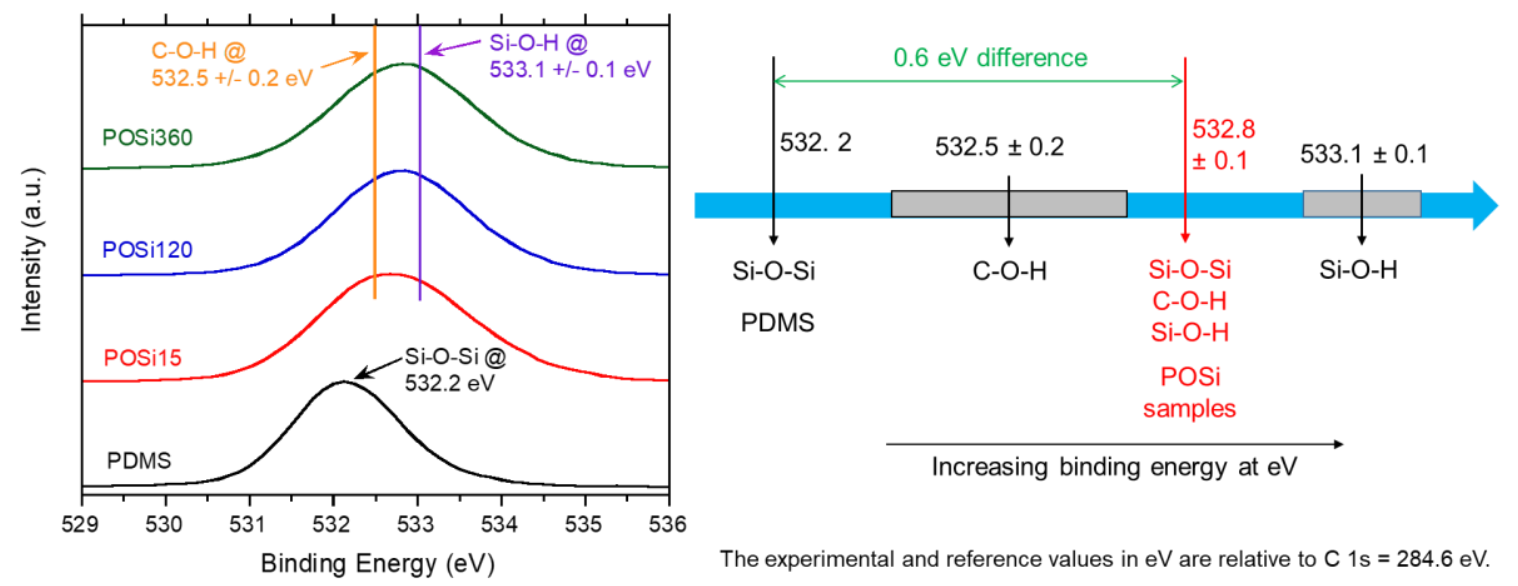

Figure S3. XPS spectra of O 1s peaks of PDMS and POSi samples. Comparison of peak positions to the right suggests the presence of $\mathrm{Si}-\mathrm{O}-\mathrm{H}$ groups.

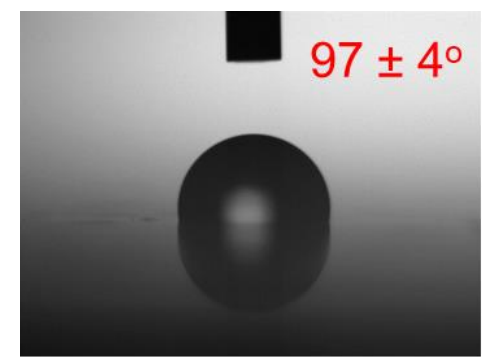

PDMS

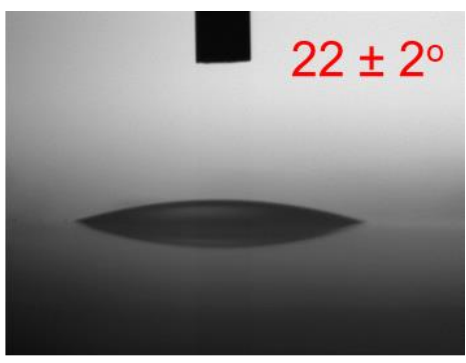

POSi120

Figure S4. Water contact angle measurement on the pristine PDMS and a POSi120 membrane.

Figure S5a compares the full range Raman spectra of the pristine PDMS and POSi120 at a Raman shift of $100-4000 \mathrm{~cm}^{-1}$. In Figure S5b and c, their high-resolution spectra are further compared, showing the same characteristic peaks for C-Si-C (160 -190 $\left.\mathrm{cm}^{-1}\right), \mathrm{Si}-\mathrm{O}-\mathrm{Si}\left(488 \mathrm{~cm}^{-1}\right), \mathrm{Si}_{-} \mathrm{CH}_{3}$ $\left(692 \mathrm{~cm}^{-1}\right)$, and $\mathrm{CH}_{3}$ stretching $\left(2907\right.$ and $\left.2966 \mathrm{~cm}^{-1}\right) .{ }^{3,4}$ Given that plasma treatment significantly changes the PDMS surface chemistry as confirmed by XPS, the identical Raman spectra of PDMS and POSi evidence that the POSi structure is too thin to be analyzed by Raman spectroscopy. For the same cause, the XRD patterns (Figure S5d) and FT-IR spectra (Figure S5e) for PDMS and POSi120 also appear to be indistinguishable, respectively. 

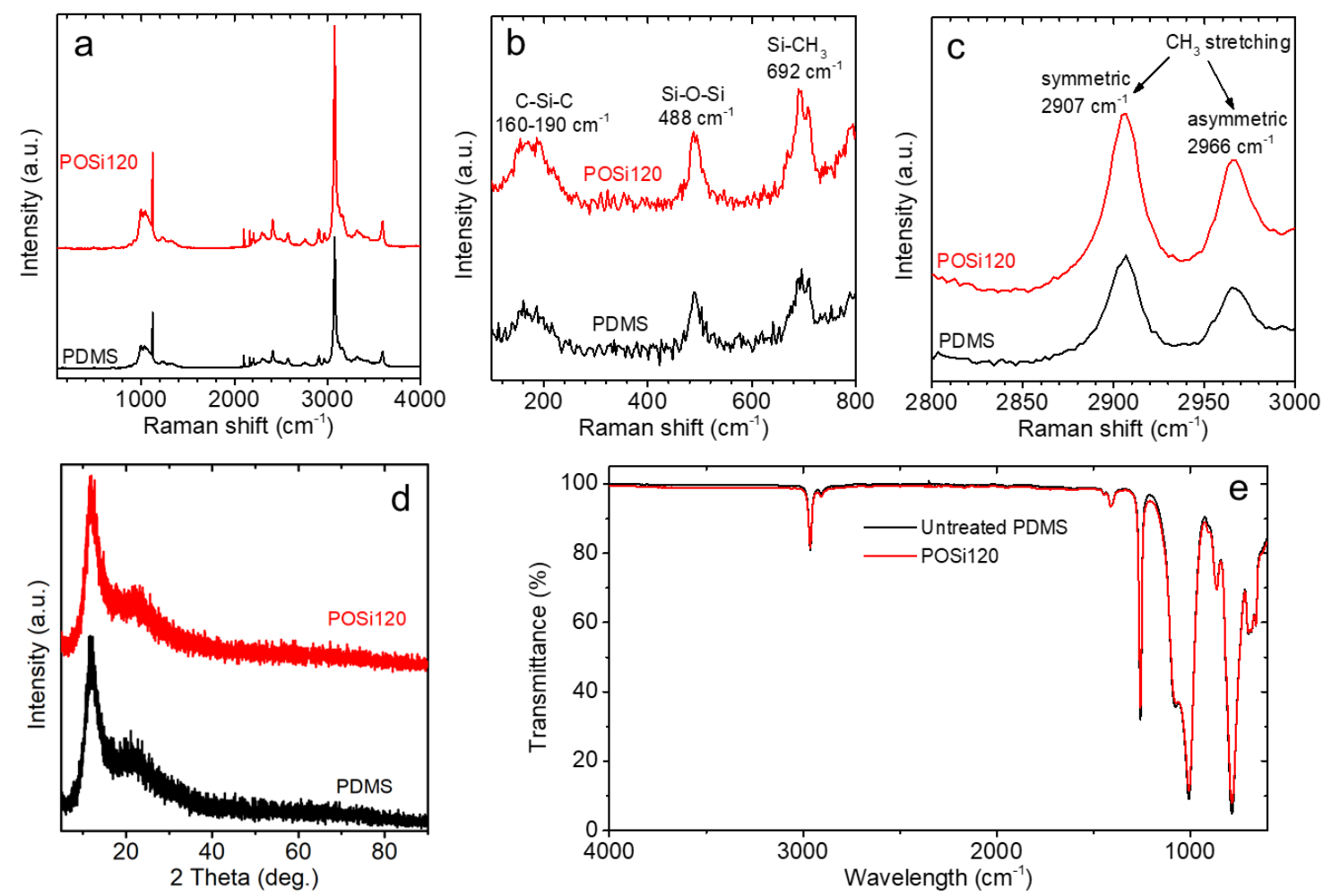

Figure S5. (a-c) Raman spectra, (d) XRD patterns, and (e) FTIR spectra of the pristine PDMS and a POSi120 membrane.

The temperature dependence of gas permeance is typically modeled by an Arrhenius equation:

$$
P / l=(P / l)_{0} \exp \left(\frac{-E_{P}}{R T}\right)
$$

where $P / l$ is the gas permeance (GPU), $(P / l)_{0}$ is the pre-exponential factor (GPU), $E_{p}$ is the activation energy of gas permeation $(\mathrm{kJ} / \mathrm{mol}), R$ is the gas constant, and $T$ is the absolute temperature $(\mathrm{K})$. As shown in Figure $\mathrm{S} 3$, the $\mathrm{H}_{2}$ and $\mathrm{CO}_{2}$ permeance in POSi120 follows this model reasonably well. The $E_{P}$ values are $33.4 \mathrm{~kJ} / \mathrm{mol}$ and $15.4 \mathrm{~kJ} / \mathrm{mol}$ for $\mathrm{H}_{2}$ and $\mathrm{CO}_{2}$, respectively.

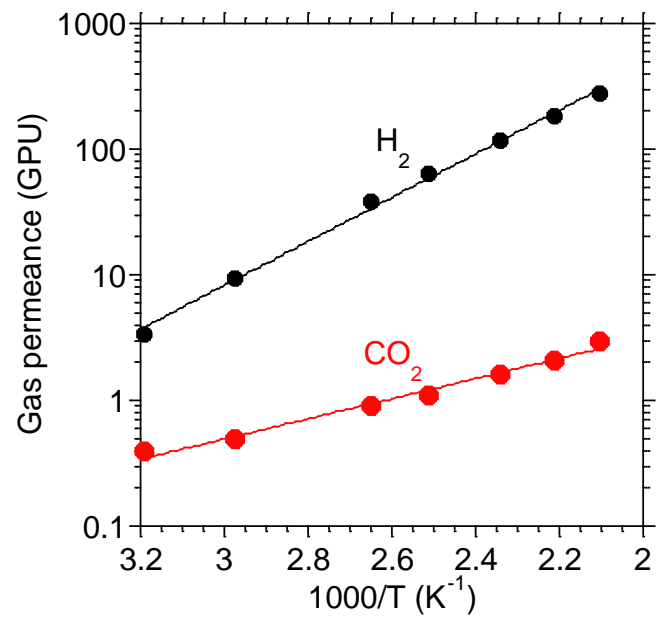

Figure S6. $\mathrm{H}_{2}$ and $\mathrm{CO}_{2}$ permeance of the POSi120 membrane as a function of test temperature. The lines are the best fits to Equation S1. 


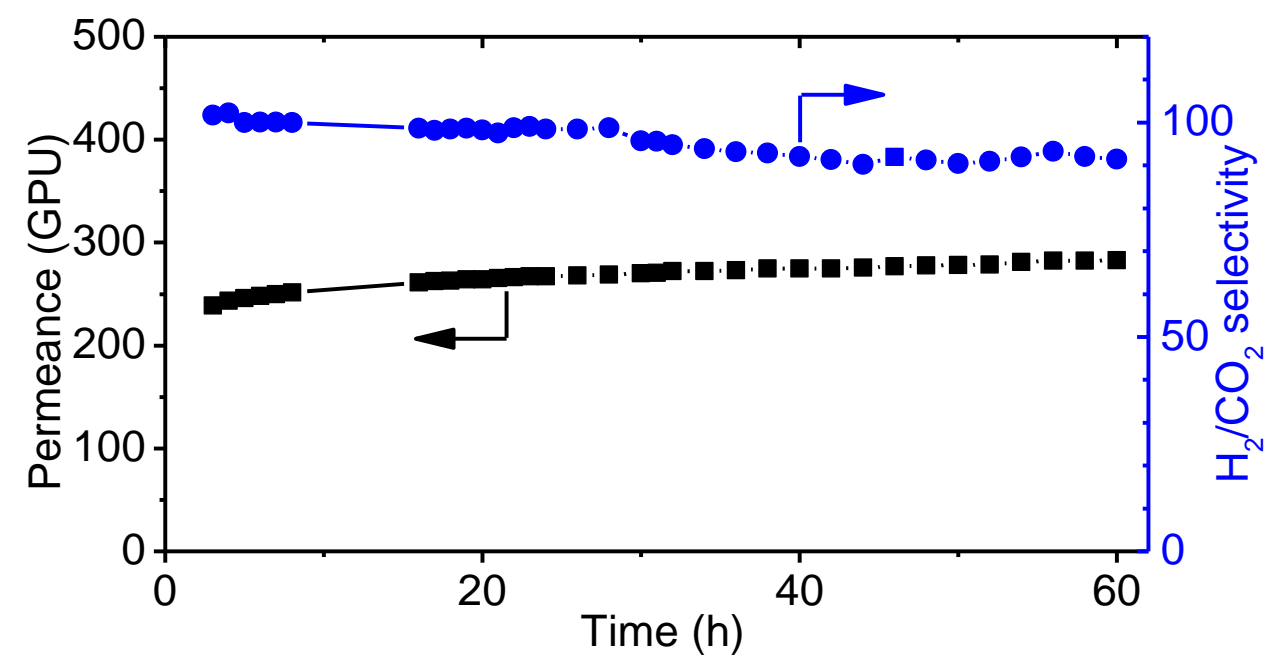

Figure S7. 60-h continuous test of a POSi120 membrane at $200{ }^{\circ} \mathrm{C}$ using a dry gas mixture of $\mathrm{H}_{2}: \mathrm{CO}_{2}(50: 50)$ after the sample was aged for 340 days.
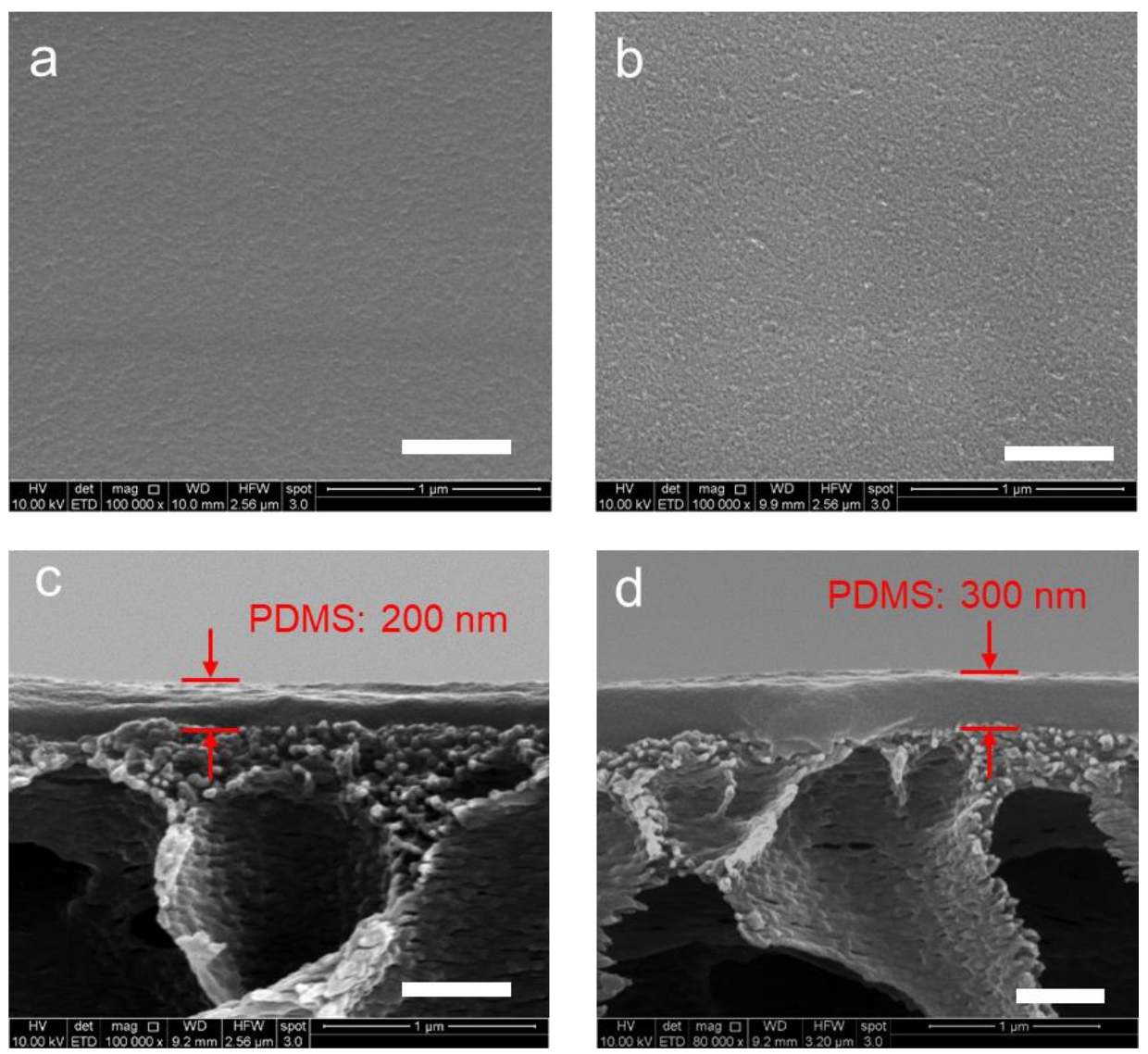

Figure S8. Surface SEM images of the PDMS TFC membranes with a PDMS thickness of (a) 1 $\mu \mathrm{m}$ and (b) $200 \mathrm{~nm}$. Cross-sectional SEM images of the PDMS TFC membranes with a PDMS thickness of (c) $200 \mathrm{~nm}$ and (d) $300 \mathrm{~nm}$. The scale bar indicates $500 \mathrm{~nm}$. 


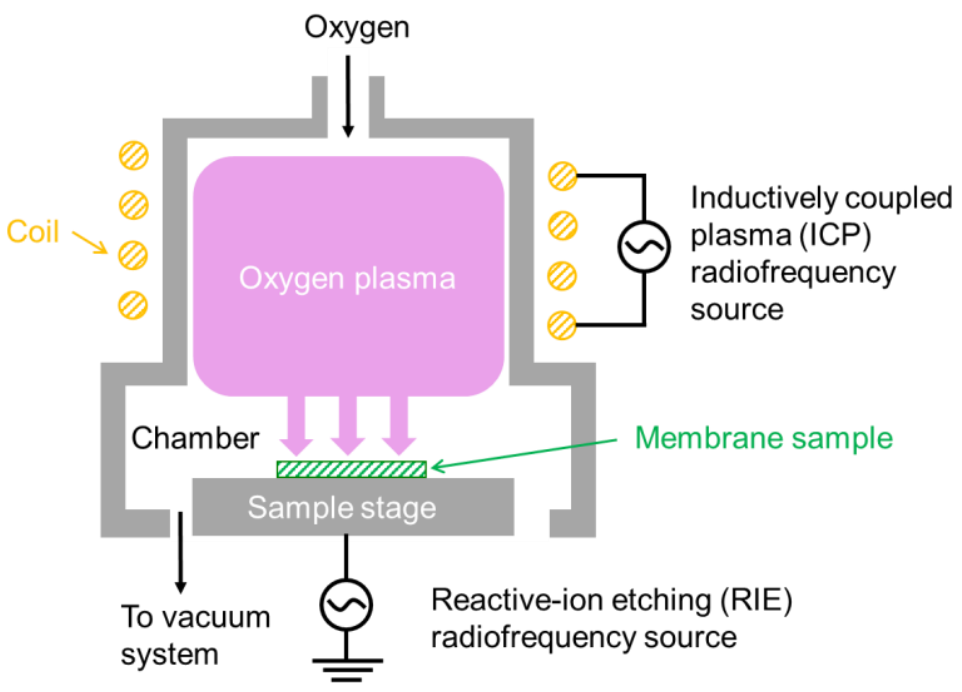

Figure S9. Schematic illustration of a Trion Technology Oracle III plasma treatment system.

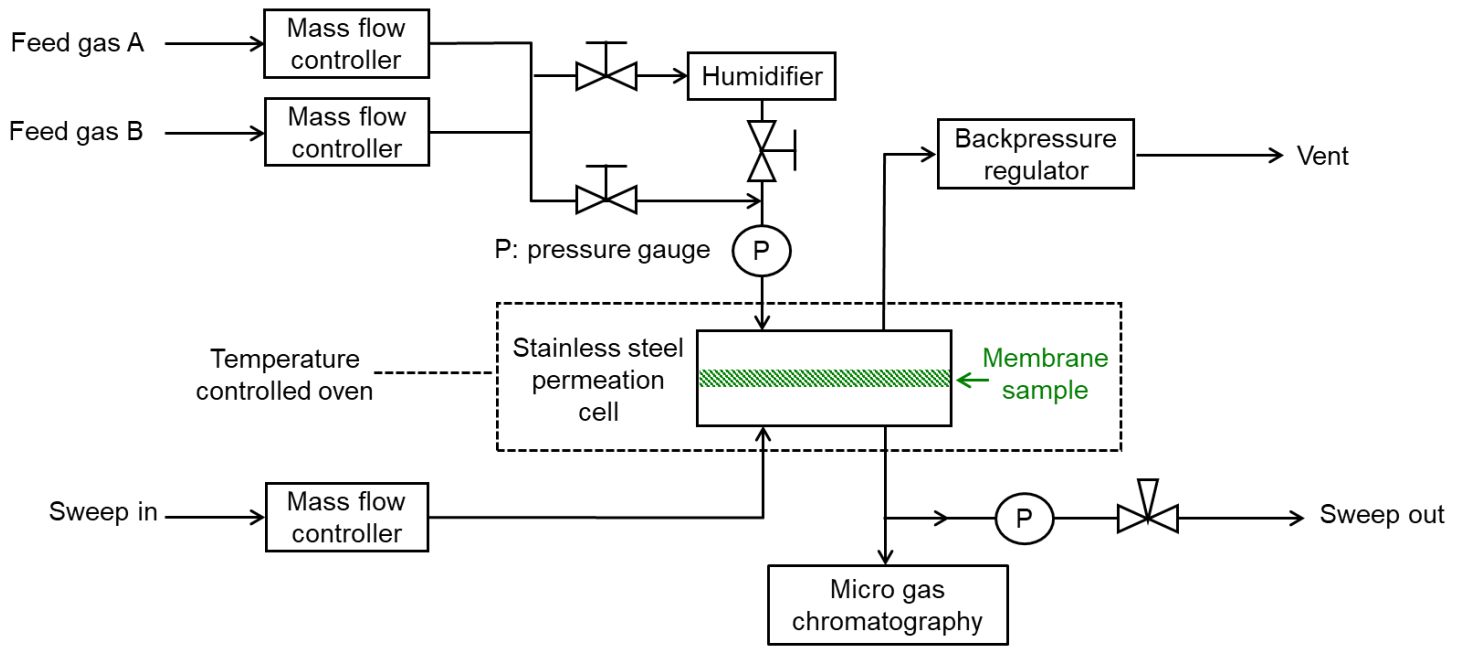

Figure S10. Schematic illustration of a constant-pressure and variable volume apparatus for pureand mixed-gas permeation measurement. 


\section{Supplementary Tables}

Table S1. Deconvoluted Si 2p and C 1s XPS peaks for the pristine PDMS and POSi samples and the calculated methyl $\left(-\mathrm{CH}_{3}\right) / \mathrm{Si}$ ratio in the POSi samples based on the deconvoluted $\mathrm{C} 1 \mathrm{~s}$ peaks and $\mathrm{C} / \mathrm{Si}$ ratio in Figure $2 \mathrm{~b}$.

\begin{tabular}{|c|c|c|c|c|c|c|}
\hline \multirow{3}{*}{ Samples } & \multicolumn{3}{|c|}{ Si $2 p$} & \multicolumn{2}{|c|}{ C 1s } & \multirow{3}{*}{$\begin{array}{c}-\mathrm{CH}_{3} / \mathrm{Si} \\
\text { ratio }\end{array}$} \\
\hline & $\begin{array}{c}\mathrm{Si}(-\mathrm{O})_{2} \\
(\%)\end{array}$ & $\begin{array}{c}\mathrm{Si}(-\mathrm{O})_{3} \\
(\%)\end{array}$ & $\begin{array}{l}\mathrm{Si}(-\mathrm{O})_{4} \text { or } \\
\mathrm{SiO}_{2}(\%)\end{array}$ & C-H (\%) & $\mathrm{C}-\mathrm{O}(\%)$ & \\
\hline & $102.1 \mathrm{eV}$ & $102.8 \mathrm{eV}$ & $103.4 \mathrm{eV}$ & $284.6 \mathrm{eV}$ & $286.0 \mathrm{eV}$ & \\
\hline PDMS & 100 & & & 100 & & 2.00 \\
\hline POSi15 & 32.3 & 31.9 & 35.8 & 83.4 & 16.6 & 0.91 \\
\hline POSi120 & 12.5 & 25.3 & 62.2 & 79.8 & 20.2 & 0.61 \\
\hline POSi360 & 9.7 & 14.9 & 75.4 & 77.1 & 22.9 & 0.53 \\
\hline
\end{tabular}

Table S2. The O/Si ratio and cumulative etching depth of the POSi samples at a given argon sputtering etch time during the XPS depth profiling.

\begin{tabular}{|c|c|c|c|c|c|c|}
\hline \multirow[b]{2}{*}{$\begin{array}{c}\text { Etch } \\
\text { time }(\mathrm{s})\end{array}$} & \multicolumn{2}{|r|}{ POSi15 } & \multicolumn{2}{|c|}{ POSi120 } & \multicolumn{2}{|c|}{ POSi360 } \\
\hline & $\begin{array}{l}\mathrm{O} / \mathrm{Si} \\
\text { ratio }\end{array}$ & $\begin{array}{l}\text { Cumulative } \\
\text { etching depth } \\
(\mathrm{nm})\end{array}$ & $\begin{array}{l}\mathrm{O} / \mathrm{Si} \\
\text { ratio }\end{array}$ & $\begin{array}{l}\text { Cumulative } \\
\text { etching depth } \\
(\mathrm{nm})\end{array}$ & $\begin{array}{l}\mathrm{O} / \mathrm{Si} \\
\text { ratio }\end{array}$ & $\begin{array}{l}\text { Cumulative } \\
\text { etching depth } \\
(\mathrm{nm})\end{array}$ \\
\hline 0 & 1.7 & 0 & 1.9 & 0 & 2.0 & 0 \\
\hline 9 & 1.6 & 1.2 & 1.8 & 0.9 & 1.9 & 0.8 \\
\hline 18 & 1.5 & 2.4 & 1.7 & 2.0 & 1.7 & 1.7 \\
\hline 27 & 1.4 & 3.9 & 1.5 & 3.2 & 1.6 & 2.9 \\
\hline 36 & 1.3 & 5.4 & 1.4 & 4.6 & 1.5 & 4.2 \\
\hline 45 & 1.2 & 7.2 & 1.3 & 6.1 & 1.3 & 5.7 \\
\hline 54 & 1.1 & 9.0 & 1.2 & 7.8 & 1.3 & 7.4 \\
\hline 63 & 1.1 & 11 & 1.2 & 9.6 & 1.2 & 9.1 \\
\hline 72 & 1.0 & 13 & 1.1 & 11 & 1.1 & 11 \\
\hline 81 & 1.0 & 15 & 1.1 & 13 & 1.1 & 13 \\
\hline 90 & 1.0 & 17 & 1.1 & 15 & 1.1 & 15 \\
\hline 99 & 1.0 & 19 & 1.0 & 17 & 1.0 & 17 \\
\hline 108 & 1.0 & 21 & 1.0 & 20 & 1.0 & 19 \\
\hline 117 & 1.0 & 23 & 1.0 & 22 & 1.0 & 21 \\
\hline 126 & 1.0 & 26 & 1.0 & 24 & 1.0 & 23 \\
\hline 135 & 1.0 & 28 & 1.0 & 26 & 1.0 & 25 \\
\hline 144 & 1.0 & 30 & 1.0 & 28 & 1.0 & 27 \\
\hline 153 & 1.0 & 32 & 1.0 & 30 & 1.0 & 29 \\
\hline 162 & 1.0 & 34 & 1.0 & 32 & 1.0 & 32 \\
\hline 171 & 1.0 & 36 & 1.0 & 34 & 1.0 & 34 \\
\hline 180 & 1.0 & 38 & 1.0 & 36 & 1.0 & 36 \\
\hline
\end{tabular}


Table S3. Gas permeance and selectivity of $1 \mu \mathrm{m}$-thick PDMS membrane samples at $35^{\circ} \mathrm{C}$ before the oxygen plasma treatment.

\begin{tabular}{|c|c|c|c|c|}
\hline \multirow{2}{*}{ Sample \# } & \multicolumn{2}{|c|}{ Gas permeance (GPU) } & \multirow{2}{*}{$\begin{array}{c}\mathrm{CO}_{2} / \mathrm{H}_{2} \\
\text { selectivity }\end{array}$} & \multirow{2}{*}{$\begin{array}{c}\mathrm{H}_{2} / \mathrm{CO}_{2} \\
\text { selectivity }\end{array}$} \\
\hline & $\mathrm{CO}_{2}$ & $\mathrm{H}_{2}$ & & \\
\hline 1 & 2269 & 576 & 3.9 & 0.26 \\
\hline 2 & 2440 & 590 & 4.1 & 0.24 \\
\hline 3 & 2290 & 556 & 4.1 & 0.24 \\
\hline Average & $2300 \pm 100$ & $570 \pm 20$ & 4.0 & 0.25 \\
\hline
\end{tabular}


Table S4 presents literature data on the $\mathrm{H}_{2} / \mathrm{CO}_{2}$ separation performance of state-of-the-art membrane materials, including polymers, mixed matrix materials, and inorganic materials. These data have also been presented in Figure 4 in the main text.

Table S4. $\mathrm{H}_{2} / \mathrm{CO}_{2}$ separation properties in selected membrane materials for comparison in Figure 4 .

\begin{tabular}{|c|c|c|c|c|c|c|c|c|}
\hline \multicolumn{2}{|l|}{ Membranes } & $\begin{array}{l}\mathrm{H}_{2}: \mathrm{CO}_{2} \text { in } \\
\text { mol\% or } \\
\text { pure gas }\end{array}$ & $\begin{array}{l}\text { Feed } \\
\text { pressure } \\
\text { (barg) }\end{array}$ & $\begin{array}{l}T \\
\left({ }^{\circ} \mathrm{C}\right)\end{array}$ & $\begin{array}{l}\mathrm{H}_{2} \\
\text { perm. } \\
\text { (GPU) }\end{array}$ & $\begin{array}{l}\mathrm{H}_{2} / \mathrm{CO}_{2} \\
\text { select. }\end{array}$ & Ref & $\begin{array}{l}\text { Ref. } \\
\# \text { in } \\
\text { the } \\
\text { text }\end{array}$ \\
\hline \multirow{2}{*}{\multicolumn{2}{|c|}{$\begin{array}{l}\text { 1. POSi120 }(1 \mu \mathrm{m} \text { PDMS }) \\
\text { 2. POSi120 ( } 1 \mu \mathrm{m} \text { PDMS }) \text { after aged for } \\
340 \text { days }\end{array}$}} & $50: 50$ & 6.9 & 150 & 144 & 61 & \multirow{5}{*}{\multicolumn{2}{|c|}{ This study }} \\
\hline & & $50: 50$ & 6.9 & 200 & 280 & 93 & & \\
\hline \multirow{3}{*}{\multicolumn{2}{|c|}{$\begin{array}{l}\text { 3. POSi120 }(\leq 1 \mu \mathrm{m} \text { PDMS }) \\
\text { 4. POSi120 (300 nm PDMS }) \\
\text { 5. POSi120 (200 nm PDMS })\end{array}$}} & $50: 50$ & 6.9 & 200 & 370 & 51 & & \\
\hline & & $50: 50$ & 6.9 & 200 & 860 & 36 & & \\
\hline & & $50: 50$ & 6.9 & 200 & 930 & 32 & & \\
\hline \multirow{7}{*}{$\begin{array}{l}\text { State-of- } \\
\text { the-art } \\
\text { membranes } \\
\text { (Figure 4a) }\end{array}$} & Proteus $^{\mathrm{TM}}$ & $58: 42$ & 50 & 150 & 300 & 32 & 5 & 6 \\
\hline & Polyamides & $50: 50$ & 7 & 140 & 350 & 50 & 6 & 46 \\
\hline & SRI PBI HFM & $50: 50$ & 10 & 200 & 100 & 40 & 7 & 9 \\
\hline & LANL PBI HFM & $63: 37$ & 13.6 & 250 & 500 & 20 & 7 & 9 \\
\hline & $\mathrm{PBI} / \mathrm{Pd}$ & $50: 50$ & 10 & 175 & 39 & 43 & 8 & 22 \\
\hline & PBI/Pd HFM & Pure & 1.0 & 60 & 80 & 10 & 9 & 48 \\
\hline & PBI/ZIF-8 & Pure & 3.5 & 180 & 64.5 & 12 & 10 & 47 \\
\hline \multirow{10}{*}{$\begin{array}{l}\text { Polymeric } \\
\text { materials if } \\
\text { fabricated } \\
\text { into } 1 \mu \mathrm{m} \\
\text { thick } \\
\text { membranes } \\
\text { (Figure 4b) }\end{array}$} & 6. $\mathrm{PBI} / \mathrm{H}_{3} \mathrm{PO}_{4}$ & $50: 50$ & 14.2 & 200 & 2.5 & 84 & 11 & 8 \\
\hline & 7. $\mathrm{PBI} / \mathrm{H}_{3} \mathrm{PO}_{4}$ & $50: 50$ & 14.2 & 200 & 19 & 32 & 11 & 8 \\
\hline & 8. PBI & $50: 50$ & 11 & 200 & 45.0 & 15 & 12 & 49 \\
\hline & 9. PBI CMS_900 & $50: 50$ & 11 & 150 & 39 & 53 & 13 & 23 \\
\hline & 10. PBI CMS $\_800$ & Pure & 7.4 & 100 & 640 & 17 & 13 & 23 \\
\hline & 11. PBI/Pd NPs $(12 \%)$ & $50: 50$ & 10 & 200 & 66 & 33 & 8 & 22 \\
\hline & 12. PI/ZIF-71 (10\%) & $50: 50$ & 7.0 & 150 & 120 & 10 & 14 & 20 \\
\hline & 13. PBI/ZIF-90 (45\%) & Pure & 3.5 & 150 & 160 & 12 & 15 & 50 \\
\hline & 14. PBI/ZIF-7 (50) & Pure & 3.5 & 180 & 440 & 17 & 16 & 21 \\
\hline & 15. PBI/ZIF-8 (30) & Pure & 3.5 & 230 & 450 & 27 & 10 & 47 \\
\hline \multirow{13}{*}{$\begin{array}{l}\text { Inorganic } \\
\text { membranes } \\
\text { (Figure 4d) }\end{array}$} & 16. Si600 & $50: 50$ & 1.0 & 200 & 1493 & 71 & 17 & 10 \\
\hline & 17. Calcined silica & Pure & 1.3 & 200 & 60 & 36.4 & 18 & 12 \\
\hline & 18. Zeolite composite & $50: 50$ & 1.48 & 200 & 209 & 20 & 19 & 13 \\
\hline & 19. RUB-15 & $50: 50$ & $\mathrm{n} / \mathrm{a}$ & 200 & 67 & 106 & 20 & 1 \\
\hline & RUB-15 & $50: 50$ & $\mathrm{n} / \mathrm{a}$ & 300 & 334 & 32 & 20 & 1 \\
\hline & 20. 2D MOF: $\mathrm{Zn}_{2}(\mathrm{Bim})_{4}$ & $50: 50$ & 1.0 & 200 & 5000 & 90 & 21 & 14 \\
\hline & 21. 2D MOF: $\mathrm{Zn}_{2}(\mathrm{Bim})_{3}$ & $50: 50$ & 1.0 & 120 & 2400 & 166 & 22 & 52 \\
\hline & 22. GO & Pure & 1.0 & 135 & 40 & 40 & 23 & 2 \\
\hline & 23. GO & $50: 50$ & 1.0 & 100 & 450 & 250 & 24 & 3 \\
\hline & 24. ZIF/GO & $50: 50$ & 1.0 & 250 & 388 & 14.9 & 25 & 53 \\
\hline & 25. $\mathrm{MoS}_{2}$ & Pure & 1.0 & 160 & 1700 & 8 & 26 & 17 \\
\hline & 26. $\mathrm{g}-\mathrm{C}_{3} \mathrm{~N}_{4}$ & Pure & 2.0 & 250 & 1450 & 10 & 27 & 18 \\
\hline & 27. iCONs & $50: 50$ & 1.2 & 150 & 2566 & 22.6 & 28 & 19 \\
\hline
\end{tabular}




\section{References}

(1) Briggs, D.; Beamson, G. XPS Studies of the Oxygen 1s and 2s Levels in a Wide Range of Functional Polymers. Anal. Chem. 1993, 65, 1517-1523.

(2) McCafferty, E.; Wightman, J. P. Determination of the Concentration of Surface Hydroxyl Groups on Metal Oxide Films by a Quantitative XPS Method. Surf. Interface Anal. 1998, 26, 549-564.

(3) Jayes, L.; Hard, A. P.; Séné, C.; Parker, S. F.; Jayasooriya, U. A. Vibrational Spectroscopic Analysis of Silicones: A Fourier Transform-Raman and Inelastic Neutron Scattering Investigation. Anal. Chem. 2003, 75, 742-746.

(4) Bae, S. C.; Lee, H.; Lin, Z.; Granick, S. Chemical Imaging in a Surface Forces Apparatus: Confocal Raman Spectroscopy of Confined Poly(dimethylsiloxane). Langmuir 2005, 21, 5685-5688.

(5) Merkel, T. C.; Zhou, M.; Baker, R. W. Carbon Dioxide Capture with Membranes at an IGCC Power Plant. J. Membr. Sci. 2012, 389, 441-450.

(6) Ali, Z.; Pacheco, F.; Litwiller, E.; Wang, Y.; Han, Y.; Pinnau, I. Ultra-Selective Defect-Free Interfacially Polymerized Molecular Sieve Thin-Film Composite Membranes for $\mathrm{H}_{2}$ Purification. $J$. Mater. Chem. A 2018, 6, 30-35.

(7) Hu, L.; Pal, S.; Nguyen, H.; Bui, V.; Lin, H. Molecularly Engineering Polymeric Membranes for $\mathrm{H}_{2} / \mathrm{CO}_{2}$ Separation at $100-300{ }^{\circ} \mathrm{C}$. J. Polym. Sci. 2020, 58, 2467-2481.

(8) Zhu, L.; Yin, D.; Qin, Y.; Konda, S.; Zhang, S.; Zhu, A.; Liu, S.; Xu, T.; Swihart, M. T.; Lin, H. Sorption-Enhanced Mixed Matrix Membranes with Facilitated Hydrogen Transport for Hydrogen Purification and $\mathrm{CO}_{2}$ Capture. Adv. Funct. Mater. 2019, 29, 1904357.

(9) Villalobos, L. F.; Hilke, R.; Akhtar, F. H.; Peinemann, K. V. Fabrication of Polybenzimidazole/Palladium Nanoparticles Hollow Fiber Membranes for Hydrogen Purification. Adv. Energy Mater. 2018, 8, 1701567.

(10) Yang, T.; Shi, G.; Chung, T. Symmetric and Asymmetric Zeolitic Imidazolate Frameworks (ZIFs)/Polybenzimidazole (PBI) Nanocomposite Membranes for Hydrogen Purification at High Temperatures. Adv. Energy Mater. 2012, 2, 1358-1367.

(11) Zhu, L.; Swihart, M. T.; Lin, H. Unprecedented Size-Sieving Ability in Polybenzimidazole Doped with Polyprotic Acids for Membrane $\mathrm{H}_{2} / \mathrm{CO}_{2}$ Separation. Energy Environ. Sci. 2018, 11, 94-100.

(12) Zhu, L.; Swihart, M. T.; Lin, H. Tightening Polybenzimidazole (PBI) Nanostructure via Chemical Cross-Linking for Membrane $\mathrm{H}_{2} / \mathrm{CO}_{2}$ Separation. J. Mater. Chem. A 2017, 5, 19914-19923.

(13) Omidvar, M.; Nguyen, H.; Huang, L.; Doherty, C. M.; Hill, A. J.; Stafford, C. M.; Feng, X.; Swihart, M. T.; Lin, H. Unexpectedly Strong Size-Sieving Ability in Carbonized Polybenzimidazole for Membrane $\mathrm{H}_{2} / \mathrm{CO}_{2}$ Separation. ACS App. Mater. Interfaces 2019, 11, 47365-47372.

(14) Japip, S.; Liao, K.; Chung, T. Molecularly Tuned Free Volume of Vapor Cross-Linked 6FDADurene/ZIF-71 MMMs for $\mathrm{H}_{2} / \mathrm{CO}_{2}$ Separation at $150{ }^{\circ} \mathrm{C}$. Adv. Mater. 2017, 29, 1603833.

(15) Yang, T. X.; Chung, T. S. Room-Temperature Synthesis of ZIF-90 Nanocrystals and the Derived Nano-Composite Membranes for Hydrogen Separation. J. Mater. Chem. A 2013, 1, 6081-6090.

(16) Yang, T.; Xiao, Y.; Chung, T. Poly-/Metal-Benzimidazole Nano-Composite Membranes for Hydrogen Purification. Energy Environ. Sci. 2011, 4, 4171-4180.

(17) de Vos, R. M.; Verweij, H. High-Selectivity, High-Flux Silica Membranes for Gas Separation. Science 1998, 279, 1710-1711.

(18) Song, H.; Wei, Y.; Qi, H. Tailoring Pore Structures to Improve the Permselectivity of Organosilica Membranes by Tuning Calcination Parameters. J. Mater. Chem. A 2017, 5, 24657-24666.

(19) Yu, M.; Funke, H. H.; Noble, R. D.; Falconer, J. L. H 2 Separation Using Defect-Free, Inorganic Composite Membranes. J. Am. Chem. Soc. 2011, 133, 1748-1750.

(20) Dakhchoune, M.; Villalobos, L. F.; Semino, R.; Liu, L. M.; Rezaei, M.; Schouwink, P.; Avalos, C. E.; Baade, P.; Wood, V.; Han, Y.; Ceriotti, M.; Agrawal, K. V. Gas-Sieving Zeolitic Membranes Fabricated by Condensation of Precursor Nanosheets. Nat. Mater. 2021, 20, 362-369.

(21) Peng, Y.; Li, Y.; Ban, Y.; Jin, H.; Jiao, W.; Liu, X.; Yang, W. Metal-Organic Framework Nanosheets as Building Blocks for Molecular Sieving Membranes. Science 2014, 346, 1356-1359. 
(22) Peng, Y.; Li, Y.; Ban, Y.; Yang, W. Two-Dimensional Metal-Organic Framework Nanosheets for Membrane-Based Gas Separation. Angew. Chem., Int. Ed. 2017, 56, 9757-9761.

(23) Kim, H.; Yoon, H.; Yoon, S.; Yoo, B.; Ahn, B.; Cho, Y.; Shin, H.; Yang, H.; Paik, U.; Kwon, S.; Choi, J.; Park, H. Selective Gas Transport through Few-Layered Graphene and Graphene Oxide Membranes. Science 2013, 342, 91-95.

(24) Li, H.; Song, Z.; Zhang, X.; Huang, Y.; Li, S.; Mao, Y.; Ploehn, H. J.; Bao, Y.; Yu, M. Ultrathin, Molecular-Sieving Graphene Oxide Membranes for Selective Hydrogen Separation. Science 2013, 342, 95-98.

(25) Huang, A.; Liu, Q.; Wang, N.; Zhu, Y.; Caro, J. Bicontinuous Zeolitic Imidazolate Framework ZIF-8@GO Membrane with Enhanced Hydrogen Selectivity. J. Am. Chem. Soc. 2014, 136, 14686-14689. (26) Achari, A.; Sahana, S.; Eswaramoorthy, M. High Performance $\mathrm{MoS}_{2}$ Membranes: Effects of Thermally Driven Phase Transition on $\mathrm{CO}_{2}$ Separation Efficiency. Energy Environ. Sci. 2016, 9, 12241228.

(27) Villalobos, L. F.; Vahdat, M. T.; Dakhchoune, M.; Nadizadeh, Z.; Mensi, M.; Oveisi, E.; Campi, D.; Marzari, N.; Agrawal, K. V. Large-Scale Synthesis of Crystalline $\mathrm{G}_{-} \mathrm{C}_{3} \mathrm{~N}_{4}$ Nanosheets and HighTemperature $\mathrm{H}_{2}$ Sieving from Assembled Films. Sci. Adv. 2020, 6, eaay9851.

(28) Ying, Y.; Tong, M.; Ning, S.; Ravi, S. K.; Peh, S. B.; Tan, S. C.; Pennycook, S. J.; Zhao, D. Ultrathin Two-Dimensional Membranes Assembled by Ionic Covalent Organic Nanosheets with Reduced Apertures for Gas Separation. J. Am. Chem. Soc. 2020, 142, 4472-4480. 